\title{
Evolutionary Algorithms Applied to Antennas and Propagation: Emerging Trends and Applications
}

\author{
Sotirios K. Goudos, ${ }^{1}$ Dimitris E. Anagnostou, ${ }^{2}$ Christos Kalialakis, ${ }^{3}$ \\ Pandian Vasant, ${ }^{4}$ and Symeon Nikolaou ${ }^{5}$ \\ ${ }^{1}$ Radiocommunications Laboratory, Department of Physics, Aristotle University of Thessaloniki, 54124 Thessaloniki, Greece \\ ${ }^{2}$ Institute of Sensors, Signals and Systems, Heriot-Watt University, Edinburgh EH14 4AS, UK \\ ${ }^{3}$ Centre Tecnologic de Telecomunicacions de Catalunya (CTTC), Barcelona, Spain \\ ${ }^{4}$ Universiti Teknologi PETRONAS, Seri Iskandar, Malaysia \\ ${ }^{5}$ Frederick University, Nicosia, Cyprus
}

Correspondence should be addressed to Sotirios K. Goudos; sgoudo@physics.auth.gr

Received 25 August 2016; Accepted 25 August 2016

Copyright (C) 2016 Sotirios K. Goudos et al. This is an open access article distributed under the Creative Commons Attribution License, which permits unrestricted use, distribution, and reproduction in any medium, provided the original work is properly cited.

Several evolutionary algorithms (EAs) have emerged in the past decades that mimic biological entities behavior and evolution. EAs are widely used for the solution of single and multiobjective optimization engineering problems. The EAs have also been applied to a variety of microwave component, antenna design, radar design, and wireless communications problems. These techniques, among others, include Genetic Algorithms (GAs), Evolution Strategies (ES), Particle Swarm Optimization (PSO), Differential Evolution (DE), and Ant Colony Optimization (ACO). In addition, new innovative algorithms that are not only biology-based but also physics-based or chemistry-based have emerged. The use of the above algorithms has an increasing impact on antenna design and propagation problems. EAs combined in several occasions with numerical methods in electromagnetics have obtained significant and successful results. Additionally, hybrid combinations of EAs are also emerging.

The papers that follow in the next pages demonstrate a range of approaches to a diverse set of application areas using different evolutionary algorithms, and with usage scenarios from radar systems to radio-astronomy applications. They represent only a fraction of the current research, the emerging trends, and applications of evolutionary algorithms. The special issue consists of nine research papers and one review paper. The algorithms used by the authors are Genetic
Algorithms (two papers), Particle Swarm Optimization (one paper), Differential Evolution (three papers), Artificial Bee Colony (one paper), Biogeography-Based Optimization (one paper), and an iterative technique (one paper). The problems addressed by the authors are single-objective (seven papers) and multiobjective (two papers). A brief description of the papers follows.

In Passive Radar Systems, the task of obtaining the mixed weak object signal against the super power signal (jamming) is challenging. C. Li et al. present a novel framework designed for weak object signal separation in the paper "A Novel Blind Source Separation Algorithm and Performance Analysis of Weak Signal Against Strong Interference in Passive Radar System.”

C. Yang et al. propose an improved artificial bee colony (ABC) algorithm called OGABC, which is based on opposition-based learning (OBL) concept and global best search equation. OGABC overcomes the shortcomings of the slow convergence rate and sinking into local optimal in the process of inversion of atmospheric duct in the paper "Investigation on the Inversion of the Atmospheric Duct Using the Artificial Bee Colony Algorithm Based on Opposition-Based Learning."

An iterative generic algorithm (GA) is proposed in the paper "Iterative GA Optimization Scheme for Synthesis of Radiation Pattern of Linear Array Antenna" by S. Todnatee 
and C. Phongcharoenpanich for the synthesis of nonuniform linear arrays.

E. Afacan uses the Differential Evolution for planar square thinned arrays design in the paper "Location Optimization for Square Array Antennas Using Differential Evolution Algorithm." The author obtains array configurations with lower peak sidelobe levels than the ones found in the literature, thus demonstrating the use of the Differential Evolution algorithm.

The application of a generic algorithm (GA) to the design of optical antennas is given by R. D. de León et al. in the paper "Evolutionary Algorithm Geometry Optimization of Optical Antennas." The authors use the finite element method (FEM) for the electromagnetic wave simulations.

Multiobjective versions of a GA and the PSO algorithm are used in the paper "Optimization of a Conical Corrugated Antenna Using Multiobjective Heuristics for RadioAstronomy Applications" for conical corrugated antenna design by S. López-Ruiz et al. The authors compare the Nondominated Sorting Particle Swarm Optimization (NSPSO) with the Nondominated Sorting Generic Algorithm (NSGAII) and conclude that NSGA-II obtains better results.

The paper "Design of UAVs-Based 3D Antenna Arrays for a Maximum Performance in Terms of Directivity and SLL" by J. Garza et al. demonstrates how to design nonuniform 3D antenna arrays when each antenna element is placed on UAVs that form a cluster. The design is carried out via Differential Evolution for Multiobjective Optimization.

Y.-F. Cheng et al. apply DE optimization for arrays' thinning and weighting in the paper "Thinning and Weighting of Planar/Conformal Arrays Considering Mutual Coupling Effects." The Active Element Pattern (a Method of Moments based technique) was utilized to take into account the mutual coupling. The efficiency of the method is demonstrated for both planar and conformal arrays.

S. K. Goudos and J. N. Sahalos apply the BiogeographyBased Optimization (BBO) algorithm with four different migration models to large thinned array design cases in the paper "Design of Large Thinned Arrays Using Different Biogeography Based Optimization Migration Models." The authors conclude that $\mathrm{BBO}$ with sinusoidal migration model generally performs better than the other algorithms.

Finally, in the review paper "Evolutionary Algorithms Applied to Antennas and Propagation: A Review of State of the Art" by S. K. Goudos et al. a survey is presented. Techniques such as the Genetic Algorithms (GAs), Particle Swarm Optimization (PSO), and the Differential Evolution (DE) are mainly reviewed along with application examples.

\section{Acknowledgments}

We would like to thank the authors who submitted their work for consideration to this special issue as well as the reviewers for their efforts and constructive criticism. The work of Christos Kalialakis was supported under EU H2020 Marie Sklodowska-Curie Grant Agreement 654734.
Christos Kalialakis

Pandian Vasant

Symeon Nikolaou

Sotirios K. Goudos 


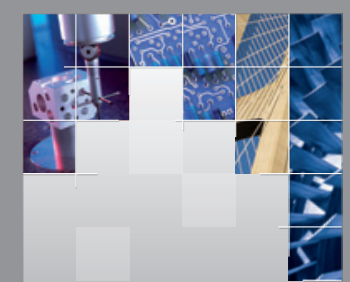

\section{Enfincering}
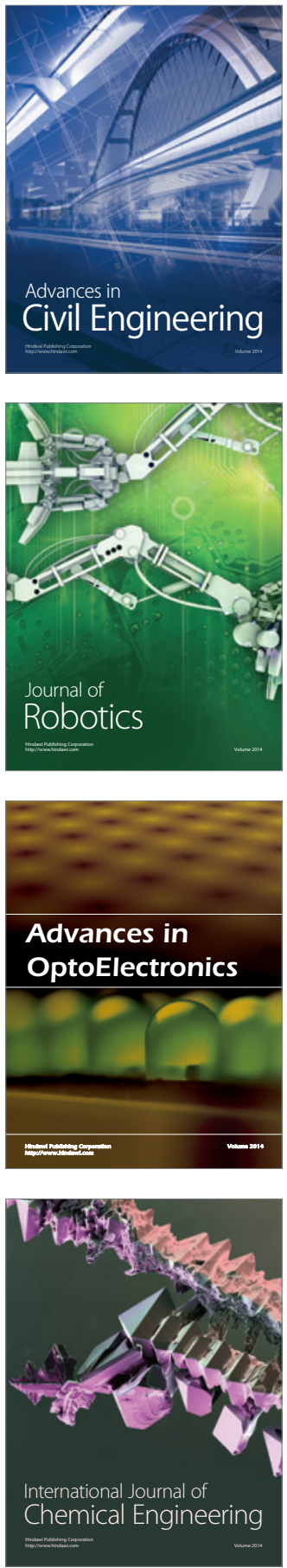

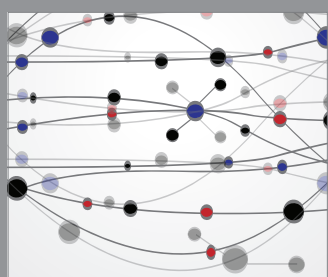

The Scientific World Journal

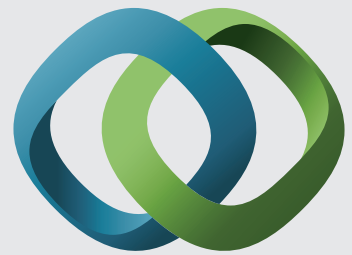

\section{Hindawi}

Submit your manuscripts at

http://www.hindawi.com
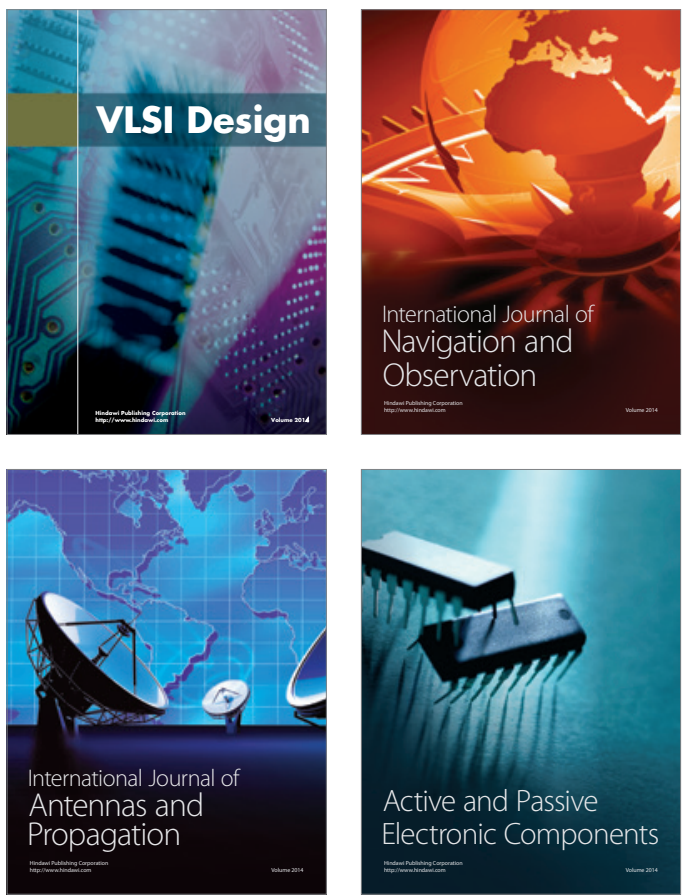
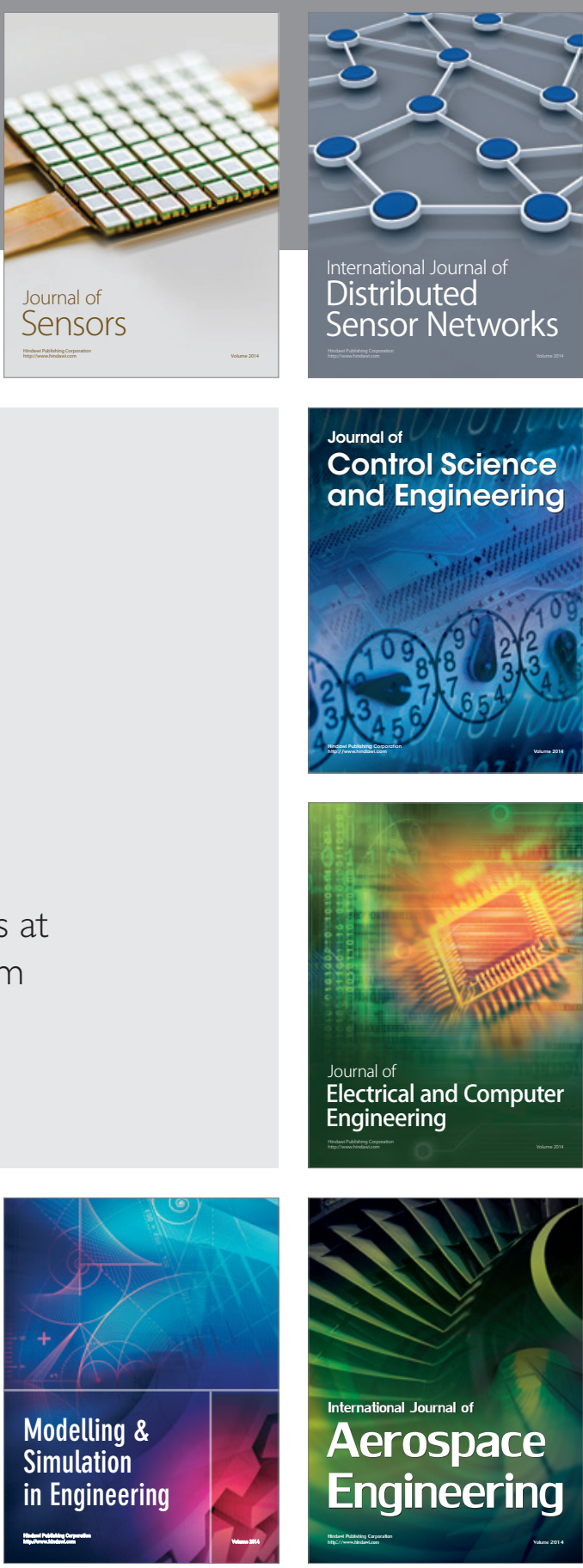

International Journal of

Distributed

Sensor Networks

Journal of

Control Science

and Engineering
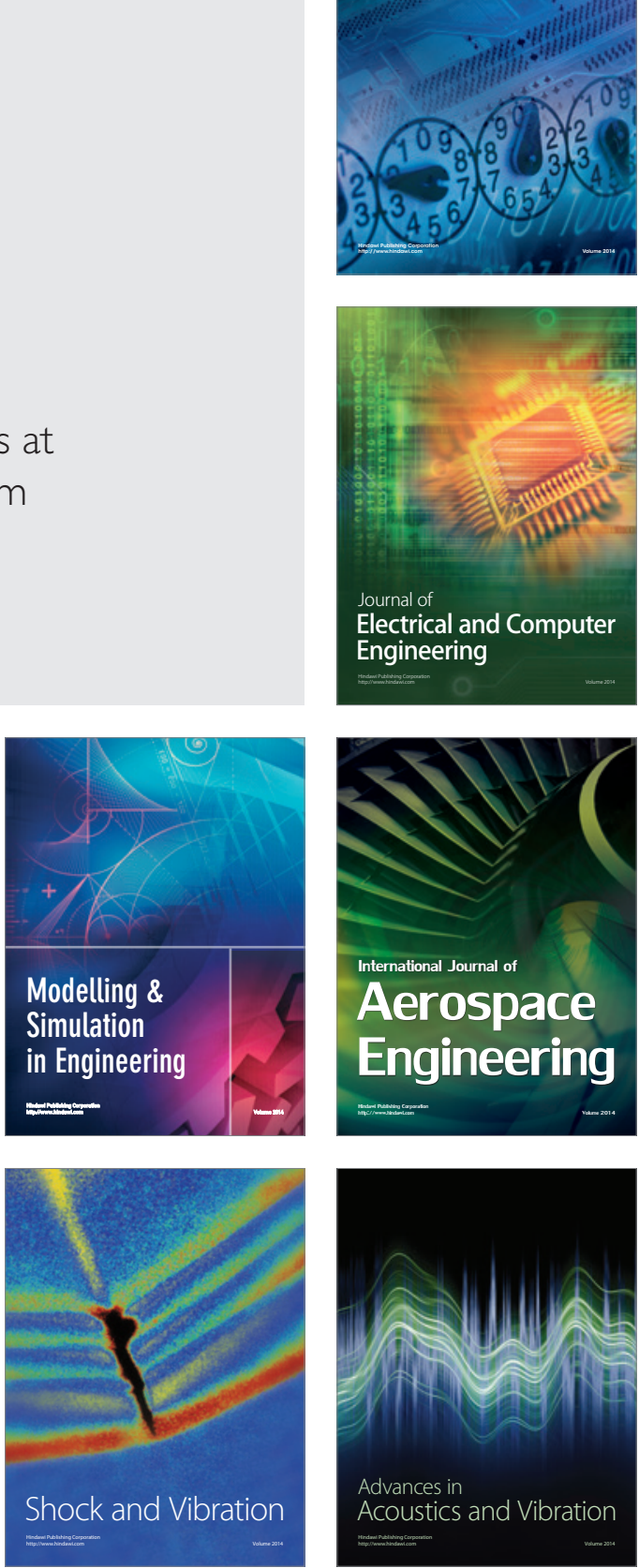